\title{
SELF DEFENSE SYSTEM FOR WOMEN SAFETY WITH LOCATION TRACKING AND SMS ALERTING THROUGH GSM NETWORK
}

\author{
B.Vijaylashmi ${ }^{1}$, Renuka.S ${ }^{2}$, Pooja Chennur ${ }^{3}$, Sharangowda.Patil ${ }^{4}$ \\ ${ }^{1}$ Research Scholar, E\&CE Department, VNEC, Shorapur - 585 220, Karnataka - India \\ ${ }^{2}$ Research Scholar, E\&CE Department, VNEC, Shorapur - 585 220, Karnataka - India \\ ${ }^{3}$ Research Scholar, E\&CE Department, VNEC, Shorapur - 585 220, Karnataka - India \\ ${ }^{4}$ Assistant Professor, E\&CE Department, VNEC, Shorapur - 585 220, Karnataka - India
}

\begin{abstract}
The world is becoming so much more unsafe for women. Social evils like molestations, dowry, crime against women, worst among all is rape is on the rise in many countries. Incidents of crime against women have been increasing at an alarming pace in Indian cities, most common incidents being rape, kidnapping, sexual harassment and eve teasing..Security for women is still a major issue as the number of crimes over women and girls is increasing day-by-day. In this age of technology, mobile phone is one gadget almost everyone uses to keep in touch with family and friends.. All they need is a device that can be carried around easily and worn whenever the woman feels unsafe.

This proposal document describes a quick responding, cost protection system for an individual and especially for women using which a woman in distress can call for help just with the press of a button on this smart gadget. Self Defense System for women safety is like a Smart Watch for Women. It has the ability to help women with technologies that are embedded into a compact device. The women wearing this device as a watch or band, in case of any harassment or when she finds that someone is going to harass, she presses a switch that is located on the watch or band or when the women has fallen the information about the attack along with the body posture and location information is sent as SMS alert to a few predefined emergency numbers And soon help is on its way! The system will consist of embedded hardware and software co-designed for this dedicated application. The system allows for knowing exact location of the individual, as soon as the trigger key on the belt is pressed. By providing the instant location of the distressed victim to the police so that the incident could be prevented and the culprit apprehended. In case if the caretaker wants to know the present location of the lady, he/she can do so by sending a SMS to the SIM number of the lady which contains a secret password. Then this system responds to such request by sending back a SMS containing location information in terms of Latitude and Longitude. This would help reduce crime against women. It also contains a shock mechanism to produce non-lethal electric shock in emergency situations to deter the attacker.
\end{abstract}

Keywords: Microcontroller, GSM, GPS, LED, ADC;

\section{INTRODUCTION}

In today's world, women safety has become a major issue as they can't step out of their house at any given time due to physical/sexual abuse and a fear of violence. Even in the 21 st century where the technology is rapidly growing and new gadgets were developed but still women and girls are facing problems. Even today in India, women cannot move at night in many places and even at day time crowded places hundreds and thousands of incidents of physical/sexual abuse happens to women every day. Among other crimes, rape is the fastest growing crime in the country today.[1]

The device descried here is a self defense system specially designed for women in distress to help them to protect themselves. This device can be fitted in a purse, belt or fitted to the girl's sandals and the panic button attached to the belt. The lady in danger can activate the system by pressing emergency button on belt or tilting her sandal. It is a simple and easy to carry device with wide range of features and functionality. [2] The basic approach is to intimate instant location and a distress message to the cops and registered number like parents, friends, media, and women cell etc. so that unfortunate incidents would be averted and to provide real time evidence for swift action against the perpetrators of crime against women. [4]

The block diagram of the conceptual system is shown in below figure. The microcontroller acts as an embedded computing system and controls the activities of all the subsystems. It is interfaced with Emergency Switch, Analog to Digital Converter (ADC), 3-Axis MEMS Accelerometer, Pressure Sensor, Body Temperature Sensor, GPS Receiver, GSM MODEM, Speech Circuit, High Voltage Shock Circuit. The microcontroller periodically monitors the status of all sensors and also keeps on checking for any incoming SMS message from the parents or any care takers. It checks for any abnormal values of body tilt, body temperature and pressure from the MEMS Accelerometer, Temperature Sensor and Pressure sensors respectively, through the ADC. If all parameters are normal and if the Emergency Switch is not pressed, it goes back in the loop and continues regular monitoring process. But, if any of these parameter values are 
abnormal or if the Emergency Switch is found to be pressed, it activates the speech circuit to make loud shouting sound to catch the attention of the nearby people for help. It also prepares the High Voltage Electric Shock Circuit to be ready to give a non-lethal shock to the attacker. [14] If the help is not available and if the system is not reset within the stipulated time, obtain location information from the GPS and prepare a text SMS containing the present location information and send SMS through GSM modem to the police control room and distress message to the preprogrammed mobile number. The design is implemented using an embedded microcontroller, in a modular form to be adaptable to different types of location tracking. Based on the total design of the system, the hardware and software of the system is designed to be near real-time monitoring of the women and immediate help. The lady can protect herself by the electric shock to the person harassing her. The software is developed in assembly language to demonstrate the system capability in providing real-time response. Using the location information supplied by this system the location using GPS and traceable through Google Maps. Thus the girl will be safe and she feels protected. At the product level it can be as compact as a mobile phone.

\section{SYSTEM ARCHIECTURE}

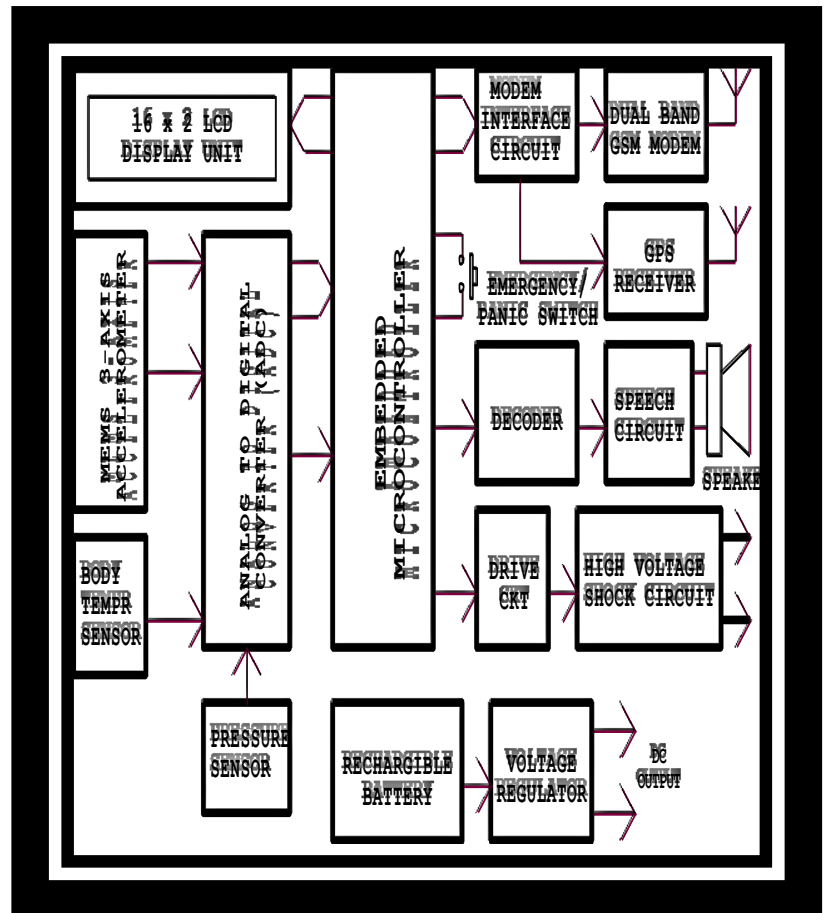

Fig.1-The block diagram of the "Self Defense System for Women Safety with Location Tracking and SMS Alerting through GSM Network".

The above figure consists of the fallowing sections.

$>$ Embedded microcontroller

$>$ Three-Axis MEMS Accelerometer

$>$ Piezoelectric Pressure Sensor

$>$ Body Temperature Sensor

$>\quad$ Analog to Digital Converter (ADC)

$>$ Clock Generator for ADC

$>$ Audio Voice Recording and Playback IC
$>$ Dual Band GSM MODEM

$>$ GPS Receiver

$>$ MODEM Interface and Level Converter

$>$ High Voltage Electric Shock Circuit

$>$ LCD display unit

$>$ Power supply

$>$ Embedded microcontroller: The microcontroller is the most important part of this system.. The microcontroller does all controlling activities of the system by executing a program stored into its flash program memory[3]. The microcontroller chosen for this project is Atmel's 89S52.it is an 8-bit microcontroller with $8-\mathrm{K}$ bytes of internal flash program memory, 256-byte data memory and 4-I/O ports. The microcontroller controls all subsystems like GSM modem, GPS receiver, MEMS Accelerometer, Pressure Sensor, Comparator and LCD panel etc. in this project.

> Three-Axis MEMS Accelerometer for Tilt (fall) Sensing: The tri-axial accelerometer sensor (ADXL335) is used to derive body posture of the subject. It is used to sense fall of the patient. It is securely fastened to the patient's body and is kept in upright position. It consists of a Three-Axis MEMS accelerometer .MEMS stands for Micro Electro Mechanical Sensor.

$>$ Piezoelectric Pressure Sensor: When the woman is subject to physical harassment, the physical pressure can be sensed using a piezoelectric sensor. A piezoelectric sensor is a device that uses the piezoelectric effect, to measure changes in pressure, acceleration, temperature, strain or force by converting them to an electrical charge.[5]

> Body Temperature Sensor and Signal Condition Circuit: The temperature sensor chosen for this project is LM35.The signal from the sensor is fed to signal condition circuit. It is for signal conditioning and standardization. It provides the required gain to the temperature signal

> Analog to Digital Converter (ADC): The ADC section is responsible to convert the analog signal into a binary number. The microcontroller can now read the digital data from ADC. An 8-bit, successive approximation type ADC is used.[7]

$>$ Clock Generator for ADC: Here we are using an IC 555 timer chip to produce these clock signals at TTL level. The IC 555 is operated in astable mode to produce square wave clock signal at about 100 $\mathrm{KHz}$ frequencies. These TTL level Clock signal is applied to clock input pin of the ADC.[6]

$>$ Audio Voice Recording and Playback IC (APR33A3 IC): The APR33A3 series are powerful audio processor along with high performance audio analog-to-digital converters (ADCs) and digital-toanalog converters (DACs). It offers true solid state storage capability and requires no software or microcontroller support. It provides high quality recording and playback with 11 minutes audio at 8 $\mathrm{KHz}$ sampling rate with 16 bit resolution. 
The APR33A3 series is specially designed for simple key trigger, user can record and playback the message averagely for $1,2,4$ or 8 voice message(s) by switch. It is suitable in simple interface or need to limit the length of single message. It can effectively reduce electric current consuming to15uA and increase the using time in any projects powered by batteries.[12]

> Dual Band GSM MODEM (Wavecom's SIM 900): This GSM Modem can accept any GSM network operator SIM card and act just like a mobile phone with its own unique phone number. It is a wireless MODEM and can send and receive data through the GSM network. It requires a SIM card and connectivity to the GSM network. It can also be used in GPRS mode to connect to the internet and use all the applications for data logging.

$>$ GPS receiver: GPS stands for Global Positioning System. Global Positioning System (GPS) is a network of satellites that continuously transmit coded information, which makes it possible to precisely identify locations on earth by measuring distance from the satellites. The purpose of using GPS module in the system is, it continuously transmits serial data like position of an individual wearing sensor, in terms of latitude and longitude, date, time and speed values to processing unit.

$>$ MODEM Interface and Level Converter: The GSM Modems and the GPS receivers work on RS 232 standard. The RS 232 standard is not compatible to TTL, therefore, it requires a line driver such as the MAX232 chip to convert RS232 voltage levels to TTL levels, and vice versa. One major advantage of the MAX232 chip is that it uses a $+5 \mathrm{~V}$ power source which is the same as the source voltage for the microcontroller.[8]

$>$ Electric Shock Circuit: The idea of implementing a small circuit which provides electric shock to the attacker is to get off the attacker and at the same time informing others about it. However, the shock intensity is so adjusted that it deters the attacker. This circuit can be carried in a purse comfortably. [14]We can provide electric shock to the attacker a no. of times. A Switch is provided in order to enable and disable the electric shock giving circuit using potentiometer VR1 you can control the intensity of shock current at the electrodes.[13]

$>$ LCD Display Unit: This system has a LCD display module for displaying various prompts and status information of the system. It is also used display the title messages and other messages while communicating with the system. A 2-line, 16 character type LCD display module is used. The microcontroller sends the signals to LCD module through its port pins. [9]

> Power Supply Unit: The power supply unit has to provide a regulated D.C supply to all sections of the system. As it is essential to operate the instrument on batteries since it is used with the person while moving. It consists of rechargeable batteries, filter capacitors and voltage regulators.
The batteries can be charged by a regular charger. Microcontroller, GSM modem, GPS receiver, MEMS Accelerometer, Pressure Sensor, Comparator and LCD panel operate on $+5 \mathrm{v}$ regulated dc voltage and Electric Shock Circuit require $12 \mathrm{v}$ dc supply. A regulated power supply unit is built to provide all above voltages at $1 \mathrm{amp}$ maximum current capacity.[10]

\section{APPLICATIONS}

$>\quad$ Can be used for the safety of women.

$>\quad$ Can be used for the safety of children.

$>\quad$ Can be used for the safety of elderly aged people.

$>\quad$ Can be used for the safety of physically challenged people.

$>\quad$ Can be used as a legal evidence of crime with exact location information for prosecution.

\section{ADVANTAGES}

$>$ Safety Device which can be carried by everyone

$>$ Ultra low power consumption.

$>$ Compact in size.

$>$ Wireless connectivity.

$>$ Easy and fast to install.

$>$ Easy Maintenance

$>$ Low cost with high performance.

$>$ Works round the clock.

$>$ Fast response.

$>$ Environmental friendly system.

\section{RESULTS AND DISCUSSION}

The system was implemented by first designing the hardware and later the software. It was rigorously tested for its proper operation and reliability. Hardware design began by designing individual circuits and their testing. Suitable modifications were carried out at various stages as necessary. After the confirmation of the proper operation of each circuitry, the Printed Circuit Board (PCB) was designed using Protel PCB making software. PCBs are fabricated by the manual process using screen printing and chemical $(\mathrm{FeCl})$ etching technique. After the holes are drilled, the mounting of components and soldering was carried out. The circuit was rigorously tested once again after mounting of all components on the PCB. Voltage levels and signals ware checked for their correctness at various stages. Some minor modifications were carried out as needed.[11]

Software design was started after the hardware was fully fabricated and tested successfully. Programs ware written in 8051 assembly language for individual modules and tested independently. After each module was tested, integration of all software modules and trouble shooting and debugging was carried out. Full functionality of the entire system with all interface modules was finally carried out to test the system for its full functionality and features. The instrument functioned as expected and the desired results were produced. 


\section{CONCLUSION}

This paper reviewed the emergency response system which is helpful for women in the incidents of crime. The key objective is to develop a low cost system which can store the data of the members in the particular locality and provide immediate alert in case of crime against women. This provides women security. Being safe and secure is the demand of the day. Our effort behind this project is to design and fabricate a gadget which is so compact in itself that provide advantage of personal security system. This device will probably be very useful for the women. It is certainly a short term and preventive solution. This will be proved as a multi-pronged strategy with the participation of multi stake holders of society. The creation of a hardware and software prototype has achieved two objectives: validation of the proposed architecture and checking whether the utilized technology is Appropriate for the system. This system will help its users in difficult situation. This system would be highly sensitive and easy to handle. Its quick action response will provide safety and security to individual user.

\section{REFERENCES}

[1] The 8051 Microcontroller and Embedded Systems using Assembly and C by Muhammad Mazidi, Janice Mazidi and Rolin McKinley.

[2] Embedded Systems by Jack Ganssle.

[3] Subrata Ghoshal, 'Embedded Systems and RobotsProjects using the 8051 Microcontroller', Cengage Learning.

[4] Microcontroller \& Embedded Systems by Ankaj Gupta.

[5] Embedded Systems Building Blocks by Jean J. Labrosse.

[6] Electronic circuit guide book - By: JOSEPH \& J. CARR

[7] Go Embedded By Yaswnt kanettkar.

[8] Embedded System Design; A Unified Hardware/Software Introduction. By Frank Vahid \& Tony Givargis.

[9] CMOS / TTL IC Data Manuals By Elector India Pvt. Ltd.

[10] 'The 8051 Microcontroller' by Kenneth J. Ayala, Cengage Learning

[11] Programming and customizing the 8051 microcontroller' by Myke Predko, TATA McGraw Hill.

[12] Architecture and Programming of 8051 Microcontrollers by Milan Verle.

[13] 8051 Microcontroller \& Embedded Systems by Rajiv Kapadia.

[14] The 8051 Microcontroller: Hardware, Software and Interfacing by James Stewart, Kai Mia. 